\title{
Michel Lagrée, Religion et modernité, France XIXe et XXe siècles
}

(Textes rassemblés par Étienne Fouilloux et Jacqueline Sainclivier et présentés par Claude Langlois), Rennes, Presses Universitaires de Rennes, 2003, 314 p. (bibliogr., tablx., illustr., cartes) (coll. « Histoire »)

\section{Muriel Pic}

\section{(2) OpenEdition}

1 Journals

Édition électronique

URL : http://journals.openedition.org/assr/2073

DOI : $10.4000 /$ assr.2073

ISSN : $1777-5825$

Éditeur

Éditions de l'EHESS

Édition imprimée

Date de publication : 1 octobre 2004

Pagination : 53-158

ISBN : 2-222-96754-6

ISSN : 0335-5985

Référence électronique

Muriel Pic, " Michel Lagrée, Religion et modernité, France XIXe et XXe siècles ", Archives de sciences sociales des religions [En ligne], 128 | octobre - décembre 2004, document 128.25, mis en ligne le 16 novembre 2005, consulté le 23 septembre 2020. URL : http://journals.openedition.org/assr/2073 ; DOI : https://doi.org/10.4000/assr.2073 
inversion des rôles, les femmes sont en prison au sortir d'une fuite en avant qui les a conduites à adopter les schémas masculins de la transgression sociale. À l'encontre des hommes, elles font peu recours au religieux et leur capacité de distance critique leur permet de mettre au clair le fait que les normes sociales qu'elles ont bafouées n'ont aucune origine sacrée. Certaines confessent que c'est la prison et ses contraintes, et non pas l'islam qui leur permet un retour sur soi. Ces sept portraits constituent les pages les plus saisissantes de ce travail qui en dernière partie affronte le rapport conflictuel entre les jeunes des banlieues et l'institution carcérale.

L'irrespect et la violence des jeunes déstabilisent les surveillants mal préparés aux comportements imprévisibles de cette sous-culture. Non formés à prendre en compte la spécificité religieuse et le sens que leur confèrent les détenus, les surveillants se barricadent derrière une culture sécularisée et laïque. Les rapports complexes renforcent les stéréotypes réciproques et alimentent les tensions quotidiennes lors du ramadan et des prières quotidiennes. Territoire laïque, la prison occulte la frontière entre religion et espace public et l'émergence du religieux comme réponse à l'anomie normative et sociale.

Le constat plus dramatique est l'absence de médiation des imams en milieu carcéral en raison de leur nombre dérisoire mais aussi de leur absence de revendications et de préparation. On regrette que les interviews soient ici peu évoquées. Les associations musulmanes brillent par leur absence et on partage le sentiment de l'auteur : « le vide institutionnel est ahurissant » (p. 251). Nul ne saurait alors s'étonner que l'islam des détenus soit un bricolage individuel qui laisse les plus désemparés à la merci du prosélytisme radical. Seule lueur à l'horizon, le profil émergent et efficace des surveillants d'origine maghrébine qui assurent les liens avec les familles avec l'aide des associations laïques et du volontariat catholique.

L'ouvrage se conclut par une réflexion sur les contradictions et ambiguïtés de la laïcité, que résume à notre avis cette phrase d'un sous-directeur : «À l'entrée nous demandons si les gens sont végétariens, s'ils mangent ou non du porc... mais nous n'osons pas demander à quelqu'un s'il est musulman» (p. 262). Voilà qui en dit long sur l'impuissance du système à maîtriser la gestion quotidienne de l'islam et la méfiance de l'institution carcérale à son égard.

Chantal Saint-Blancat.
Religion et modernité, France $\mathrm{XIX}^{\mathrm{e}}$ et $\mathrm{XX}^{\mathrm{e}}$ siècles. (textes rassemblés par Étienne Fouilloux et Jacqueline Sainclivier et présentés par Claude Langlois), Rennes, Presses Universitaires de Rennes, 2003, 314 p. (bibliogr., tablx., illustr., cartes) (coll. « Histoire »).

Comment l'historien du religieux se place-til vis-à-vis de la foi ? Doit-il justifier de sa « faiblesse de croire »? Interroger la relation de l'historien à son objet d'étude est une démarche intellectuelle indispensable pour M.L., chercheur et professeur d'histoire contemporaine à Rennes, décédé prématurément en 2001 : «l'historien croit toujours parler des autres, jamais de lui-même; pourtant ses lecteurs le retrouvent dans ses livres. » M.L. condamne tout autant «l'agnosticisme méthodologique » que la «confessionnalité rampante et inavouée » de l'historien du religieux à l'endroit de son objet. Il relève le pari de concilier une croyance religieuse et une scientificité laïque, et incarne le paradoxe qui fonde sa recherche c'est-à-dire celui d'une époque où la religion côtoie les nouveautés techniques, où la science rencontre la foi au prix d'un choc propre à la modernité. On est alors tenté, avec Claude Langlois, chargé de présenter ce recueil posthume, de placer M.L. dans la filiation de Michelet et De Certeau bien que la notoriété de sa prose ne soit pas celle de ses illustres prédécesseurs. Le présent ouvrage, «Religion et modernité », nous permet de découvrir celle-ci à travers une vingtaine d'articles organisés autour des trois pôles de recherche de l'auteur: le premier temps est consacré à des textes s'interrogeant sur la discipline même qu'est l'histoire religieuse moderne. Puis, un ensemble d'articles interroge les rites religieux et leurs survivances spatiales et temporelles en Bretagne. Enfin une dernière série porte sur la percée de la technique dans le monde catholique et, inversement, sur l'influence du religieux dans les processus de modernisation.

Mais si l'A. s'interroge sur le «modus loquendi » de l'historien du religieux dans le monde moderne et contemporain, il ne perd pas de vue que cette question implique également de définir la discipline qu'il pratique. Née avec la crise d'identité de la religion chrétienne en Occident, la nouveauté qu'a été pour le $\mathrm{XX}^{\mathrm{e}}$ siècle l'histoire religieuse s'explique par la distinction laïque de cette dernière vis-à-vis de la théologie et de l'histoire ecclésiastique. L'histoire religieuse s'inscrit pour M.L. au centre de ce qu'il désigne comme histoire culturelle, aux côtés de l'ethnographie, des sciences du langage et surtout de la sociologie. Le mot d'ordre méthodologique est alors le comparatisme 
qualitatif, celui-ci étant efficace à deux niveaux : tout d'abord, il permet de confronter des phénomènes similaires entre des zones géographiques différentes (la Bretagne et certaines régions de la Suisse et de l'Irlande). Ensuite, il autorise un travail entre les disciplines : l'A. utilise les sciences du langage dont les instruments d'analyse lui semblent encore bien précaires - pour étudier le « Mandement de Carême » à l'aune de la dichotomie entre énoncé et énonciation. Il a également recours, continuellement, à une approche sociologique du fait religieux, justifiée par l'analyse de l'influence de Durkheim, Weber et Troeltsch sur la question, et lui permettant de comparer par exemple la manifestation de rue et le rite de la procession. Ainsi, au fil de ses articles, le lecteur voit le postulat de M.L. s'affermir selon lequel la religion tient un rôle fondamental dans la constitution du lien social.

Dès lors ce n'est plus seulement d'une histoire de l'institution religieuse dont il est question dans l'historiographie moderne mais bien davantage d'une étude de la religion populaire et de son influence dans les mentalités collectives. Durant les périodes de crise, selon l'A., ce sont les dévotions archaïques qui viennent suppléer au défaut de la religion officielle en transcendant les oppositions politiques. En Bretagne par exemple, région souvent marginalisée à cause de la centralisation, le religieux revitalise le lien social par la création d'une contre-société ou d'une subculture. Pour désigner ces réactions sociales spontanées, par lesquelles se forment notamment des bastions catholiques cimentant la population, M.L. emploie une expression pour le moins moderne, celle de « choc religieux ».

Or, le choc, au temps du déclin de la civilisation rurale et de la transformation économique du pays par la technisation, se manifeste aussi dans la création d'alliances inédites entre le religieux et la modernité. Si Garcia Moreno (18211875), président de l'Équateur, est tout à la fois dictateur et saint martyr, l'idée de la confession par téléphone ou la vision du curé de campagne en effraction de vitesse dans une voiture de course a aussi de quoi surprendre. Et ce sont justement ces réalités du monde religieux moderne qu'étudie M.L. : le rôle du sport dans la volonté du catholicisme moderne de retenir les hommes dans la carrière religieuse, l'influence de l'Église dans le développement de la technique, à travers un vulgarisateur scientifique comme le Père Moigno, au XIX ${ }^{\mathrm{e}}$ siècle, ou encore les liens entre le cinéma et le monde catholique. L'opposition philosophique séculaire entre nature et culture retrouve toute son actualité dans les discours entourant par exemple l'invention du bateau à vapeur, tandis que l'intégration de la technique dans le quotidien des hommes religieux est analysée jusque dans les textes canoniques avec, par exemple, l'insertion d'un «benedicto vehiculi seu curus $»$. À cette introduction de certains objets modernes au sein de rites religieux, en l'occurrence la bénédiction de chemins de fer ou d'automobiles, répond la dissolution de l'objet religieux dans l'histoire culturelle via de multiples métaphores telles que «mythes politiques et religions séculières, dieux du stade, du spectacle, de la bande dessinée ». Propres à une époque où s'intensifie l'immigration et se multiplient les rencontres avec «l'autre religieux », ces processus de métaphorisation touchent également les représentations du diable. En effet, au même titre que les nouveautés machinistes de la modernité, la présence d'altérités religieuses différentes du christianisme participe, en Occident, aux « renouvellements successifs de l'Antéchrist et des forces du Mal ».

Ainsi, le présent ouvrage s'ouvre sur un article à vocation confessionnelle où 1'A. y explique son double rapport à la foi et à la science, et s'achève sur une étude du démoniaque, intime à chacun en ses multiples visages. Configuration éditoriale qui, en hommage à M.L., montre bien comment pour ce dernier, l'écriture de l'histoire n'aura été ni une question personnelle ni une réponse objective, mais une recherche nourrie par l'expérience singulière de «l'historien qui, à force de fréquenter les morts, les retrouve vivants derrière la poussière des archives, acquiert une familiarité avec eux. »

Muriel Pic.

\subsection{LAIGNEL-LAVASTINE (Alexandra).}

\section{Cioran, Éliade, Ionesco. L'oubli du fascisme.} Paris, PUF, 2002, 553 p. (coll. «Perspectives critiques »).

Voici un ouvrage impressionnant, par la richesse de sa documentation et la rigueur de ses analyses. Travaillant sur des écrits en langue roumaine inconnus en France et sur des archives jusqu'ici inexploitées, A.L.-L. montre l'ampleur de l'engagement fasciste et antisémite d'Emil Cioran et de Mircea Éliade dans la Roumanie des années 1930 et 1940. Eugène Ionesco ne partageait pas du tout leurs options, mais par une sorte de «solidarité anticommuniste de l'exil», il acceptera de faire le silence sur le passé de ses deux illustres compatriotes. 